\title{
Throughput improvement for cell edge users
}

\author{
${ }^{1}$ Pinal N. Parlekar, ${ }^{2}$ Nirmal S. Kothari, ${ }^{3}$ Prof. Mary Grace Shajan \\ ${ }^{1,3}$ Dept.of Electronics \& communication Engg. PIEL, Limda Vadodara, India \\ ${ }^{2}$ Dept.of Electronics \& Telecommunication Engg. R.C. Patel Institute Of Technology Shirpur,India
}

\begin{abstract}
Users at the cell edge in a cellular network suffer from low data rate due to low SINR Cooperative transmission schemes which are used in wireless networks to improve the spectral efficiency. In this paper, we study the downlink capacity of edge users in a cellular network and see whether base station cooperation improves the spectral efficiency. The base-stations coordinate their transmission to the two cell-edge users in order to improve their Signal-to-interference-noise ratio (SINR) and throughput. Selective Cooperation, where the selection criterion is based on throughput, is proposed. The capacity achieved through Cooperation is shared equally among the cell-edge users. Results show that, the proposed hybrid scheme provides a better result compared to full-time cooperation.
\end{abstract}

Keywords-spectral efficiency, data rate,cell edge user,Capacity

\section{Introduction}

There is ever increasing demand to support higher data rates for broadband services like triple play, online gaming etc., over wireless networks, which requires a large capacity.

However, with scarcity of available radio resources, to achieve a good capacity and Quality of Service (QoS) efficient utilization of channel resources is important. In a conventional cellular network, a terminal receives signals not only from the base station of that cell, but also from other cell base stations.

Using a proper frequency reuse, such interference is reduced to a tolerable limit. However, this method of using different frequency bands for different cells will decrease the spectral efficiency. In a full frequency reuse network, this interference degrades the system performance, and thereby reduces network capacity.

Using Base Station Cooperation, this ability to receive signals from multiple base stations can be utilized as an opportunity to improve the spectral efficiency of the cellular network and achieve higher data rates for cell edge users. Cooperative transmission utilizes the inherent user diversity available in a multi-user environment to provide higher spectral efficiency [1-3]. In [1] and [3], cooperation among active users for the uplink channel in wireless networks is described. The active users under cooperation have its own information to transmit, and therefore, do not simply act as a mobile relay stations. Since the inter-user link is also a noisy channel, there is a possibility that the information received by a user from the other user is corrupted. In [3], coded cooperation is proposed where each user decodes the signal of the other user that needs to be relayed, and will relay only if it is successfully decoded. In case of unsuccessful decoding, the users go to non-cooperative mode. In [2], cooperative strategies like amplify-forward and decode-forward for adhoc or per-to-peer wireless networks are proposed In [4], it is shown that the downlink efficiency can be improved using Coherent Coordinated transmission (CCT) from multiple base stations. Two types of coordination transmission are proposed, namely, Equal Rate using Zero Forcing and Equal Rate Using Dirty Paper Coding. In Equal Rate using Zero Forcing, the transmission from all base stations intended for a particular user do not interfere with other users. In the Dirty Paper Coding scheme, knowledge of the interference is used at the transmitter for coding.

Comparison of different coordination schemes like full coordination, partial coordination and no coordination is presented in [5] for a downlink Multiple Input Multiple Output (MIMO) system in a slow fading channel. In the full coordination scheme, the transmit covariance matrix for all the possible downlink channels between base stations and the users is computed using Dirty Paper Coding by a central coordinator to provide maximum sum throughput, based on the Channel Quality Information (CQI) provided by the base stations. These covariance matrices are then sent to corresponding base stations. However, this entire process adds significant latency. A new partial coordination scheme, where the base stations transmit in Time Division Multiple Access (TDMA) mode is proposed in [5]. In the allotted slot, each base station transmits to its associated users using Space Division Multiple Access (SDMA). Cooperative encoding and scheduling in a Networked MIMO system is discussed in [6], in order to suppress Other Cell Interference (OCI) and thereby achieve maximum capacity in MIMO downlink channel.

In [7], it is shown that in a multi-cell environment, using cooperation the overall interference can be reduced only marginally, whereas the interference within the cooperation region is largely reduced. This leads to a question whether it is worth doing cooperation all the time, i.e., whether the performance gains are worth the cost addition in terms of the extra complexity added in the signal processing to perform cooperation. 
In this paper, we analyze the cooperation scenario in a multi cell environment where the other cell interference is significant. The capacity achieved through cooperation is shared equally among the cell-edge users, i.e., resources are shared fairly among the cooperating users. The transmission rate to each user is determined based on the signal to interference plus noise ratio (SINR). Cooperative transmission by two base stations can improve this SINR by transmitting jointly to one user at a time. However, the increase in terms of throughput may not always be enough to increase the throughput of each of the users. In such a scenario, we propose a selective cooperation scheme based on user throughput that provides better capacity than full cooperation. The downlink environment under consideration will not have any interference from users in the same cell. They are properly separated in time, frequency or code such that orthogonality exists. Inter-cell interference is allowed by doing a full frequency re-use in each cell.

The rest of the paper is organized as follows: Section 2 describes the system model, signal to interference noise ratio (SINR) and user throughput with and without cooperation. Section 3 describes the SINR for different modes of Cooperation considered in this paper. Section 4 presents the cooperation selection algorithm and an example for UMTS. Section 5 presents the simulation results and conclusions are presented in section 6 .

\section{System Model}

The basic system model and transmission protocol is as shown in Figure 1. Base stations BS1 and BS2 are the candidates for cooperation, to transmit signals to mobile terminals MS1 and MS2. For BS1, BS2 is one of the interfering base stations among the total 12 base stations in a re-use 1 network.

More than one base station can be involved in cooperation, but for simplicity we are considering only two stations to form a coalition. The observation still holds good even for three station coalition. The signals from the serving BS and from the neighbor BS arrives at the terminal at the same time, i.e., received signal by the terminal from the two base stations are frame synchronized. The frame duration in which the BS1 transmits to MS1 is divided into two sub-frames,

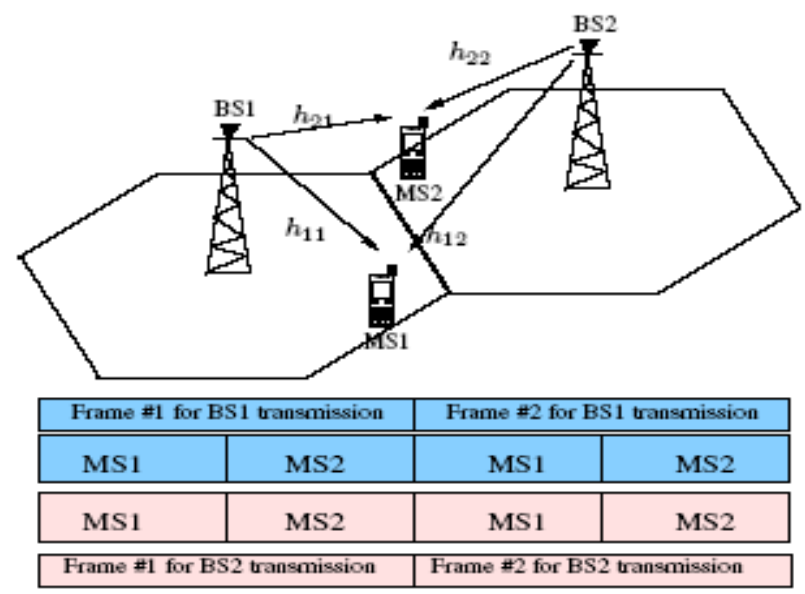

Fig. 1: System Model

Where the first sub-frame is used for signal transmission to MS1 and the second one to MS2. Similarly, BS2, which is under cooperation with BS1, transmits in the same sequence of BS1.

The received signals at MS1 and MS2 is y1 and y2, and is given by system equation 1, where hij is the channel between terminal $\mathrm{i}$ and BS $\mathrm{j} . \mathrm{x} 1$ is transmit signal of BS1 and $\mathrm{x} 2$ is that of BS2.zi is the total interference received by MS $\mathrm{i}$ due to transmissions from all the base stations other than the one under cooperation (in this case BS2) and ni is the additive white Gaussian noise.

\section{A. No Cooperation}

$$
\left[\begin{array}{l}
y_{1} \\
y_{2}
\end{array}\right]=\left[\begin{array}{ll}
h_{11} & h_{12} \\
h_{21} & h_{22}
\end{array}\right]\left[\begin{array}{l}
x_{1} \\
x_{2}
\end{array}\right]+\left[\begin{array}{l}
z_{1} \\
z_{2}
\end{array}\right]+\left[\begin{array}{l}
n_{1} \\
n_{2}
\end{array}\right]
$$

Under normal operation that is when there is no cooperative transmission, the signal to interference noise ratio (SINR) in the downlink for MS1 is given by

$$
\operatorname{SINR}_{\mathrm{nc}}=\frac{\left|h_{11}\right|^{2} E\left\{X_{1}^{2}\right\}}{\sigma_{n}^{2}+\sum_{k=2}^{12}\left|h_{1 k}\right|^{2} E\left\{X_{i}^{2}\right\}}
$$

Where hij represents the channel between the terminal $i$ and base station $j, E\left\{X^{2}{ }_{i}\right\}$ is the average transmit power of Base Station $\mathrm{i}$, and $\sigma_{\mathrm{n}}^{2}$ is noise variance. 
The capacity (or throughput) for terminal MS1 in bits/sec/Hz can derive from the Shannon Capacity as

$$
C_{\mathrm{nc}}=\log _{2}\left(1+b \mathrm{SINR}_{\mathrm{nc}}\right)
$$

Where, $b$ is determined by the SNR gap between the practical coding scheme and the theoretical limit.

\section{B. Cooperation}

When terminal MS1 is in cooperation with BS1 and BS2, SINRcoop, SINR of the downlink channel will depend on the type of cooperation scheme. The capacity (or throughput) for terminal MS1 under cooperation in bits/sec/Hz will be

$$
C_{\text {coop }}=\alpha \log _{2}\left(1+b \text { SINR }_{\text {coop }}\right)
$$

The factor $\alpha$ in eq. 4 defines the proportion of resource sharing among the terminals under cooperation. In our system, considering resource fairness, the value for $\alpha$ is $1 / 2$.

\section{Cooperation Selection}

Under the resource fairness constraint, the users in the serving cell and the neighbor cell who decided to cooperate for an SINR improvement will share the available resource (time, frequency or code) between them equally. Therefore, the individual user throughput is $1 / 2$ of the actual capacity of the cooperative transmission as in (4). Considering $b=1$ in the capacity expressions (3) and (4), for a low SINR regime, as $\log (1+x)=x$, for the user capacity in "Cooperation mode" to be at least equal to what the same user could achieve under "No cooperation", the SINR in the former must be twice of the latter, i.e., should be $>=3 \mathrm{~dB}$.

The exact expression for the capacity (or user throughput) for cooperative scheme with resource constraint, to perform better than normal transmission, i.e., Ccoop > Cnc is shown below:

$$
\begin{aligned}
& \frac{1}{2} \log \left(1+b \operatorname{SINR}_{\text {coop }}\right)>\log \left(1+b \operatorname{SINR}_{\mathbf{c}}\right) \\
& 1+b \operatorname{SINR}_{\text {coop }}>\left(1+b \operatorname{SINR}_{\mathrm{nc}}\right)^{2} \\
& 1+b \operatorname{SINR}_{\cos p}>1+b^{2} \operatorname{SINR}_{\mathbf{a c}}^{2}+2 b \operatorname{SINR}_{\mathrm{nc}} \\
& \operatorname{SINR}_{\text {coop }}>b \mathrm{SINR}_{\mathrm{nc}}{ }^{2}+2 \mathrm{SINR}_{\mathrm{nc}}
\end{aligned}
$$

From the expression (8), for low SINR regime, our earlier approximation is valid. However, in the high SINR regime, the relationship between the two SINR is not linear, rather it is exponential. Even though, the SINR under cooperation (SINRcoop) is always better than the normal SINR (SINRnc), the user throughput of former is not always better than the latter. Hence, it is worthwhile, for the user to decide whether to perform cooperation in the downlink channel.

A brief description of the selection algorithm is given in Algorithm 1. This selection algorithm is of low complexity as it is approximation of the exact expression presented in (8) with $b=1$. The user decides on cooperation with the measurements of its own channel and the nearest neighbor.

The decision is informed to the base station of the serving cell.

The serving station informs the neighbor station whether to do cooperation or not with a single bit information based on the input from the user.

\section{Simulation And Results}

A 19 cell full re-use multi-cell environment is simulated based on Monte Carlo methods to analyze the performance of user capacity and SINR for three transmission scenarios namely, i) Without Cooperation, ii) With Cooperation and iii) Selective Cooperation. Selective Cooperation is a hybrid scheme, where cooperative transmission is performed only if the (4) is greater than (3) as described in algorithm 1.

A cellular network of radius $500 \mathrm{~m}$, operating at $1800 \mathrm{MHz}$ with one cell edge user per cell is considered for simulations.

The channel gains for both signal and interference are based on COST-231 path loss model [10] including fading and lognormal shadowing. The correction factors for the path loss model are that of metropolitan/urban areas. The shadowing component is a Gaussian random variable with zero mean and $10 \mathrm{~dB}$ of standard deviation. Fading component is an iid random variable with zero mean and unit variance. The transmission power of each base station (at the antenna) is $2 \mathrm{~W}(33 \mathrm{dBm})$. The superposition of signals for cooperation is performed in three different ways as mentioned in section 3 . 
Our observation from simulation revealed that with probability 0.45 , the user throughput without cooperation (3) is better than (4) for $\alpha=1 / 2$. Since, cooperation in a multi-cellular environment with full resource fairness is advantageous only half the time, it is better to do a hybrid transmission of both normal operation and cooperation that can give a better user throughput. Average throughput and SINR for cell edge user for different cooperative schemes is shown in Table I and II. Averaging is done over 105 frames for each combination of cooperative scheme and selection of cooperation. The observed values from the simulation given in the table, clearly shows the advantage of selective cooperation over full cooperation. Even though, the average SINR of Scheme 2 with cooperation is same as Scheme 1 with Selective cooperation, the capacity of the latter is better than the former. User throughput captured over 1000 frames for scheme 1 for full cooperation and selective cooperation is shown in Fig. 3. Throughput captured for first hundred frames is captured and shown in Fig.4, which depicts the fact that there are crossovers in user throughput for with and without cooperation. Hence, selective cooperation is a better option to get maximum throughput.

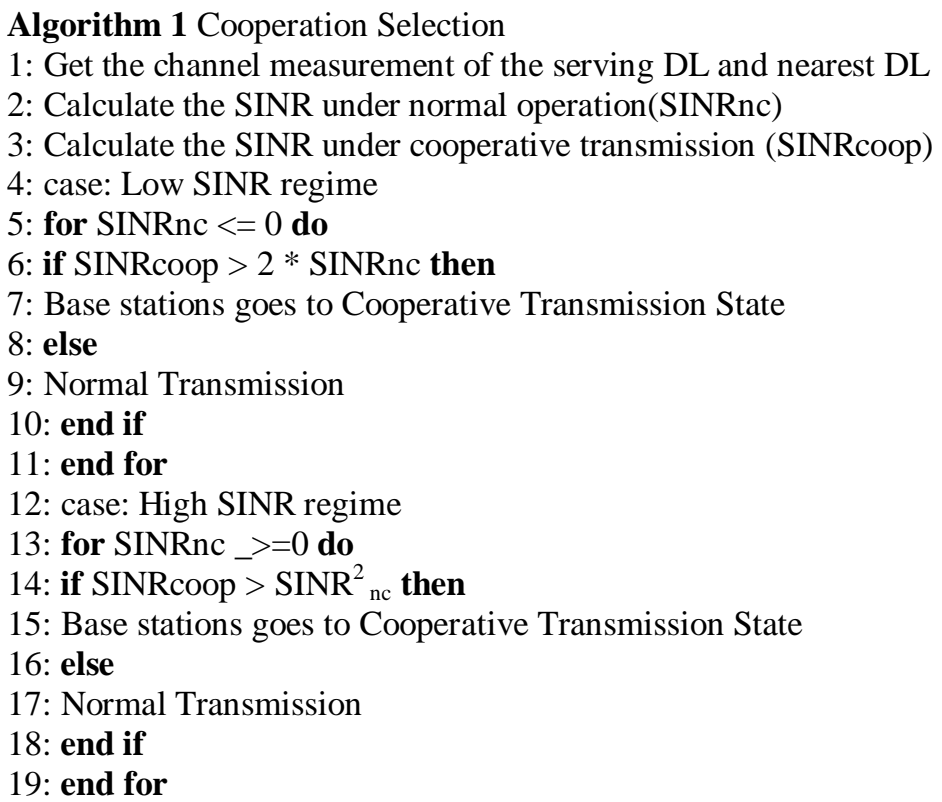

TABLE I: Average Throughput for cell Edge user (bits/sec/Hz) for different Cooperation schemes

\begin{tabular}{|l|c|c|c|}
\hline Type of Schemes & Scheme 1 & Scheme 2 & Scheme 3 \\
\hline Without Cooperation & 1.034 & 1.034 & 1.034 \\
\hline With Cocperation & 1.235 & 1.197 & 1.347 \\
\hline Selective Cooperation & 1.596 & 1.582 & 1.674 \\
\hline
\end{tabular}

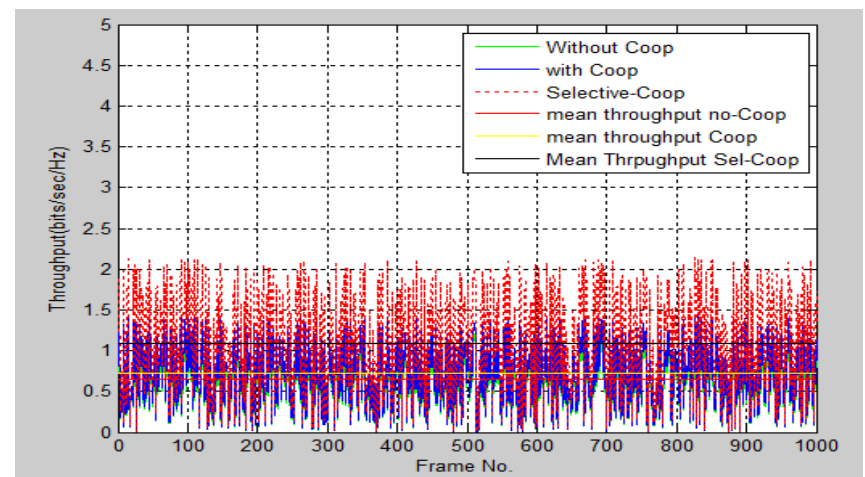

Fig. 3: User Throughput Comparison for Various Operations in Scheme 1

TABLE II: SINR of cell Edge user (dB) for different Cooperation schemes

\begin{tabular}{|l|c|c|c|}
\hline Type of Schemes & Scheme 1 & Scheme 2 & Scheme 3 \\
\hline \hline Without Cocperation & -7.50 & -7.50 & -7.50 \\
\hline With Cooperation & 3.52 & 2.79 & 4.70 \\
\hline Selective Cocperation & 2.79 & 2.58 & 3.88 \\
\hline
\end{tabular}




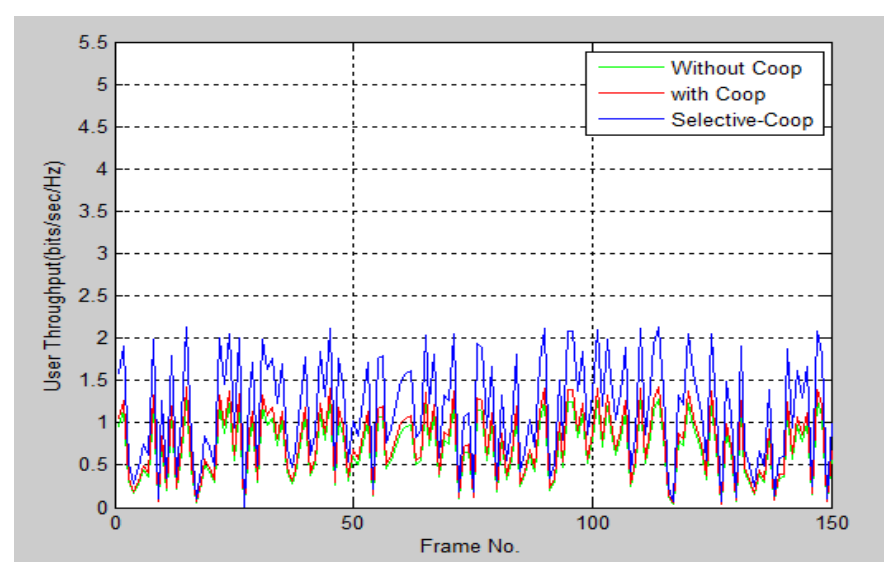

Fig.4: Snapshot of User Throughput for Various Operations in Scheme 1

\section{Conclusions}

In this paper, we presented simulation analysis of downlink cooperation in a multi-cell cellular network. In resource fairness cooperation, the user capacity of a cell-edge user is not always better than normal transmission. The simulation results show that for almost half the time user capacity with cooperation is poorer than the capacity with normal operation.

By doing a selective cooperation, both capacity and SINR is improved. The throughput improvement is about $33.3 \%$ from full cooperation to selective cooperation for same SINR.

\section{REFERENCES}

[1] A. Sendonaris, E. Erkip and B. Aazhang, "User Cooperation Diversity - Part I System Descriptiuon," IEEE Transactions on Communications, vol. 51, no. 11, pp.1927-1938, Nov 2003.

[2] J. N. Laneman, G. W. Wornell and D. N. C. Tse,"An efficient protocol for realizing cooperative diversity in wireless networks," in Proc. IEEE ISIT 2001, p.294, Washington, D. C., June 2001.

[3] A. Nosratinia, T. E. Hunter and A. Hedayat, "Cooperative Communication in Wireless Networks," IEEE Communications Magazine, pp. 74-80, Oct 2004.

[4] G. J. Foschini, H. Huang, K. Karakayali, R. A. Valenzuela and S. Venkatesan“, "The Value of Coherent Base Station Coordination", Proceeding of 2005 CISS; The John Hopkins University, March 16-18, 2005.

[5] T. Tamaki, K. Seong and J. M. Cioffi,"Downlink MIMO Systems Using Cooperation among Base Stations in a Slow Fading Channel", Proceeding of IEEE International Conf. on Communications 2007, pp. 4728-4733, June 2007.

[6] J. G. Andrews, W. Choi and R. W. Heath Jr, "Overcoming Interference in Spatial Multiplexing MIMO Cellular Networks", IEEE Wireless Communications Magazine, vol. 14, no. 6, pp.95-104, Dec 2007.

[7] CollaborativeMIMO",http://www.ieee802.org/16/tgm/contrib/C80216m-07-244r1.doc

[8] J. Akhtar and D. Gesbert, "Extending Orthogonal Block Codes with partial feedback", IEEE Transactions on Wireless Communications, vol.3, no. 6, pp.1959-1962, Nov 2004.

[9] H. Holma and A. Toskala, HSDPA/HSUPA for UMTS: High Speed Radio Access for Mobile Communications. John Wiley \& Sons, 2006.

[10] Urban Transmission Loss Models for Mobile Radio in the 900 and 1800 MHz bands, EURO-COST 231 Std. 